J. Warren

Nagoya Math. J.

Vol. 51 (1973), 191-208

\title{
ORDER OF FUNCTIONS BOUNDED ON A SPIRAL
}

\author{
JOSEPH WARREN
}

\section{Section 1. Introduction}

The purpose of this paper is to improve a result of Schnitzer and Seidel [6] and to continue an analogy between entire functions and the class of functions which are holomorphic and unbounded in the unit disk, but bounded on a boundary spiral. The existence of such functions was established by Valiron [7].

The author wishes to thank Prof. W. Seidel for his help in this paper.

Throughout this paper the unit disk $|z|<1$ in the $z$-plane shall be denoted by $D$ and the upper half-plane $\mathscr{I}(\zeta)>0$ in the $\zeta$-plane shall be denoted by $H$ where $\mathscr{I}(\zeta)$ and $\mathscr{R}(\zeta)$ stand for the imaginary and real part of $\zeta$ respectively. The maximum of the modulus of the function $f(z)$ on the circle $|z|=r$ shall be denoted by $M(r, f)$.

DeFinition 1. A spiral in $D$ is a set $S=\{z(t) \mid 0 \leq t<1\}$ where $z(t)$ continuous and one-to-one on $0 \leq t<1,0<|z(t)|<1$ on $0<t<1$, and $\lim _{t \rightarrow 1}|z(t)|=1$. Moreover, for each branch, $\arg z(t)$, of the argument of $z(t), 0<t<1$, it is required that either $\lim _{t \rightarrow 1} \arg z(t)=+\infty$ or $\lim _{t \rightarrow 1} \arg$ $z(t)=-\infty$.

The class of functions which are holomorphic and unbounded in $D$, but bounded on the spiral $S$ shall be denoted by $\mathscr{V}(S)$.

Some analogies will now be given between the class $\mathscr{V}(S)$ and the class of entire functions.

Every non-constant entire function has infinity as an asymptotic value. Valiron obtained the result that every function in $\mathscr{V}(S)$ has infinity as an asymptotic value [7].

The Picard theorem asserts that a non-constant entire function assumes every complex number with at most one exception in each neighborhood of the boundary point infinity. It is known that each $f \in \mathscr{V}(S)$

Received March 31, 1971. 
assumes every complex number infinitely often with at most one exception in the disk $D$ [2, Theorem 1]. It is true that $f$ assumes every complex number with at most one exception in each neighborhood of each boundary point.

The analogies obtained in this paper will be between the order of growth of functions in $\mathscr{V}(S)$ and the order of growth of entire functions. In particular, analogies to Wiman's theorem [3, p. 39] and the AhlforsDenjoy Theorem are found [1]. Before stating these analogies it is necessary to introduce some preliminary notions.

Let $D$ be the unit disk and $S$ be a spiral in $D$. Some assumptions can be made about the spiral near $z(0)$ which are convenient. Let $z(0)=0$

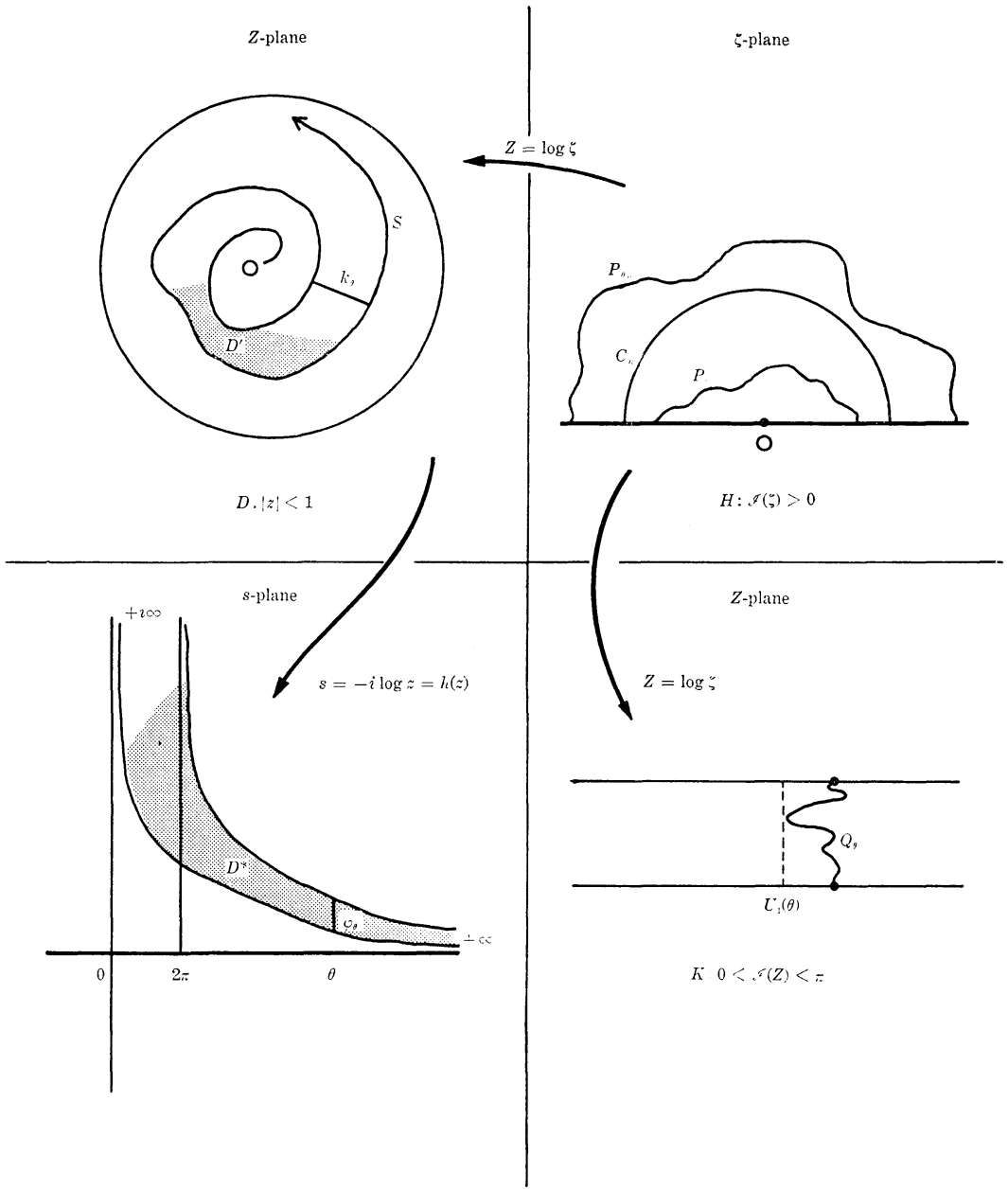

Fig. 1 
and let $\lim _{t \rightarrow 1} \arg z(t)=+\infty$ for each branch of the argument of $z(t)$. Let $\lim _{t \rightarrow 0} \arg z(t)$ exist for each such branch. With these conventions let the symbol $\arg z(t)$ stand for that branch of the argument of $z(t)$ for which $0 \leq \lim _{t \rightarrow 0} \arg z(t)<2 \pi$. In addition, suppose that $\arg z(t)>0$ for $t>0$. These assumptions are allowable because the shape of the initial part of the spiral is irrelevant to the results in this paper.

Let $S$ be a spiral in $D$ with the above restrictions. The set $D^{\prime}=$ $D-S$ is a simply connected region. There is a largest interval $(0, a)$, $a>0$, contained in $D^{\prime}$. For any $x$ on this segment let $h(x)=2 \pi$ $+i(-\log x)$ where $-\log x$ is real. From this determination let $s=h(z)=$ $-i \log z$ be continued throughout $D^{\prime}$ and a one-to-one conformal mapping $s=\theta+i \tau=h(z)$ is obtained. Let $D^{*}=h\left(D^{\prime}\right)$. Denote the boundary point of $D^{*}$ which corresponds to 0 under $s=h(z)$ by $+i \infty$ and the boundary point of $D^{*}$ which corresponds to the prime end of $D^{\prime},|z|=1$, under $s=h(z)$ by $+\infty$ (see Figure 1).

Definition 2. Let $D, S, D^{\prime}$ and $D^{*}$ be defined as above. Let $\phi_{\theta}$ be the unique vertical straight line crosscut of $D^{*}$ above $s=\theta, \theta>2 \pi$, which separates $+i \infty$ from $+\infty$ in the sense of Ahlfors [1, pp. 5-6]. Let $k_{\theta}$ be the inverse image of $\phi_{\theta}$ under $s=h(z)$. Let $k(\theta)$ and $\varphi(\theta)$ be the lengths of the straight line segments $k_{\theta}$ and $\varphi_{\theta}$, respectively.

Throughout this paper the symbols just introduced shall retain their meaning.

Definition 3. For $f \in \mathscr{V}(S)$ define $K(\theta)=\sup _{z \in k \theta}|f(z)|$ and, for $\theta_{0}>2 \pi$, let

$$
\lambda(S)=\lim _{\theta \rightarrow \infty} \frac{\log \log K(\theta)}{\pi \int_{\theta_{0}}^{\theta} \frac{d t}{k(t)}},
$$

and

$$
\rho(S)=\varlimsup_{\theta \rightarrow \infty} \frac{\log \log K(\theta)}{\pi \int_{\theta_{0}}^{\theta} \frac{d t}{k(t)}}
$$

It is easy to see that both $\lambda(S)$ and $\rho(S)$ do not depend on the lower limit of the integral. The lemma below follows from straight forward limit arguments and the fact that 


$$
\lim _{x \rightarrow 0} \frac{\log (1+x)}{x}=1
$$

From part (3) it follows that $k(t)$ in both expressions of Definition 3 may be replaced by $\phi(t)$.

LemMA 1. For $t>2 \pi$, and for $\varphi(t)$ and $k(t)$ defined as in Definition 2 relative to the spiral $S$ in $D$, the following relations hold. If $k(t)=$ $x-y$, then $\varphi(t)=\log x-\log y$ and

$$
\begin{aligned}
& \text { (a) } \varphi(t)>k(t), \\
& \text { (b) } \lim _{t \rightarrow \infty} \frac{k(t)}{\varphi(t)}=1, \\
& \text { (c) } \lim _{\theta \rightarrow \infty} \frac{\int_{\theta_{0}}^{\theta} \frac{d t}{\varphi(t)}}{\int_{\theta_{0}}^{\theta} \frac{d t}{k(t)}}=1 .
\end{aligned}
$$

If the lower order relative to $S$ of a function $f \in \mathscr{V}(S)$ is defined to be $1 / 2 \lambda(S)$ then it shall be proved in Section 2 , Theorem 1 that the lower order is not less than $1 / 2$.

Wiman's theorem states that non-constant entire function which is bounded on a half line has lower order not less than 1/2 [3].

Thus it is seen that the analogy of class $\mathscr{V}(S)$ to entire functions extends to notions of order.

In Section 3 the analogue to the Ahlfors-Denjoy theorem [1] will be obtained. In Section 4 the analogue to the extension of the AhlforsDenjoy theorem proved by MacIntyre [4] will be obtained.

Section 2 contains a proof that the theorem of Schnitzer and Seidel follows from the analogue to Wiman's theorem. (See the corollary to Theorem 1).

Section 2. Wiman's Theorem in $\mathscr{V}(S)$.

All the notations and conventions developed in Section 1 shall be freely used in this section. Two more conformal transformations are needed.

Let $z=g(\zeta)$ be a conformal transformation mapping the upper half plane $H: \mathscr{I}(\zeta)>0$ onto the region $D^{\prime}$ which is contained in the unit 
disk $|z|<1$. Let $g(0)=0$ and the boundary point of $H$ at infinity correspond to the prime end, $|z|=1$, of $D^{\prime}$. Although $z=g(\zeta)$ is not fully normalized it is to be considered in any given situation as a fixed mapping.

Map the half-plane $H$ onto the strip $K: 0<\mathscr{I}(Z)<\pi$ in the $Z$-plane by the mapping $Z=\log \tau$. In the sequel the following notations shall be adhered to. Let $P_{\theta}$ and $Q_{\theta}$ be the images of $k_{\theta}$ in $H$ and $K$, respectively, under the appropriate combined mappings (See Figure 1). For $\theta>2 \pi$, let

$$
U_{1}(\theta)=\operatorname{Inf}_{Z \in Q \theta} \mathscr{R}(Z)
$$

It is now possible to prove the analogue to Wiman's theorem.

THEOREM 1. Let $f \in \mathscr{V}(S)$, then $\lambda(S) \geq 1$.

Proof. By Ahlfors' First Fundamental Theorem [1, p. 10], there exists a $\theta^{\prime}>2 \pi$ and a $\theta_{1}^{\prime}>\theta^{\prime}$ such that for $\theta>\theta_{1}^{\prime}$,

$$
U_{1}(\theta)>\pi \int_{\theta^{\prime}}^{\theta} \frac{d t}{\varphi(t)}
$$

Assume, contrary to the theorem, that $\lambda(S)<1$. Suppose that $\theta_{0}=\theta^{\prime}$ and, by Lemma 1 , replace $k(t)$ by $\varphi(t)$ in the definition of $\lambda(S)$. Then

$$
\lim _{\theta \rightarrow \infty} \frac{\log \log K(\theta)}{\pi \int_{\theta^{\prime}}^{\theta} \frac{d t}{\varphi(t)}}<1
$$

There exists a sequence $\theta_{1}, \theta_{2}, \cdots$, with $\theta_{1}^{\prime}<\theta_{1}<\theta_{2}<\cdots$, and a $\gamma$, $0<\gamma<1$, such that

$$
\log \log K\left(\theta_{n}\right)<\pi \gamma \int_{\theta^{\prime}}^{\theta_{n}} \frac{d t}{\varphi(t)}, \quad n=1,2, \cdots
$$

From (5)

$$
\pi \int_{\theta^{\prime}}^{\theta_{n}} \frac{d t}{\varphi(t)}<U_{1}\left(\theta_{n}\right), \quad n=1,2, \cdots
$$

Let $C_{n}$ be the semicircle in the half-plane $H: \mathscr{I}(\zeta)>0$ with radius $\exp \left[\pi \int_{\theta^{\prime}}^{\theta_{n}} \frac{d t}{\varphi(t)}\right]$. By the continuity and normalization of the conformal 
transformations and (8), the set $P_{\theta_{n}}$ is outside of $C_{n}$ and so $P_{\theta_{n}}$ tends uniformly to $\infty$ as $n \rightarrow \infty$.

Let $F(\zeta)=f(g(\zeta))$, then $F(\zeta)$ is holomorphic in $H$ and is continuous and bounded on the real axis $\mathscr{I}(\zeta)=0$. It is clear that $K\left(\theta_{n}\right)=\sup _{\zeta \in P \theta n}|F(\zeta)|$.

Let $\zeta$ be an arbitrary point of $P_{\theta_{n}}$. Then, from (7) and (8),

$$
\begin{aligned}
|F(\zeta)| & \leq \sup _{\zeta^{\prime} \in P_{\theta}}\left|F\left(\zeta^{\prime}\right)\right|=K\left(\theta_{n}\right) \\
& \leq \exp \left\{\exp \left[\pi \lambda \int_{\theta^{\prime}}^{\theta_{n}} \frac{d t}{\varphi(t)}\right]\right\} \\
& \leq \exp \left\{\exp \left[U_{1}\left(\theta_{n}\right)\right]\right\}^{r} \\
& \leq \exp \left[|\zeta|^{r}\right] .
\end{aligned}
$$

The function $F(\zeta)$ satisfies all the conditions of the Phragmén-Lindelöf Principle [5] and so is bounded. But because $f \in \mathscr{V}(S), F(\zeta)$ cannot be bounded. This contradiction establishes Theorem 1.

COROLlARY. Schnitzer and Seidel [6]. Let $r=a(t)$ be a continuous strictly increasing function in $0 \leq t<\infty$ such that $0 \leq a(t)<1, a(0)=0$, and $\lim _{t \rightarrow \infty} a(t)=1$. Let $a=\lambda(r)$ be the inverse function of $r=a(t)$. Setting $S=\left\{z(t) \mid z(t)=a(t) e^{i t}, 0<t<\infty\right\}$ let $f \in \mathscr{V}(S)$. Then the following relation holds:

$$
\frac{\lim }{r \rightarrow 1} \frac{\log \log M(r, f)}{\lambda(r)}=\infty
$$

Proof. With the notation and hypotheses of the corollary, $k(\theta)=$ $a(\theta)-a(\theta-2 \pi)$ is continuous and positive. Thus L'Hospital's rule applied to

$$
R(\theta)=\frac{\pi \int_{\theta_{0}}^{\theta} \frac{d t}{k(t)}}{\theta}
$$

implies

$$
\lim _{\theta \rightarrow \infty} R(\theta)=\infty
$$

Because $k_{\theta}$ is contained in $|z| \leq a^{\prime}(\theta)$, the inequality $M(r, f) \geq K(\lambda(r))$ holds. These two facts and a direct elementary argument using Theorem 1 give the corollary. 
Remark. It is seen that Theorem 1 is a generalization of the corollary in two ways. First, it applies to unrestricted spirals. Second, in the case of spirals for which the corollary applies, Theorem 1 implies the corollary.

Remark. The corollary states that the growth of $M(r, f)$ depends on the growth of $\lambda(r)$. If $r=a(\theta)$ grows rapidly to 1 , then $\lambda(r)$ grows slowly to $\infty$ and, consequently, $M(r, f)$ is allowed to grow more slowly. By considering the following example, and by applying Theorem 1 , it is seen that not only is the rate that $r=a(\theta)$ tends to 1 involved but also the manner in which $a(\theta)$ tends to 1 .

EXAMPLE. Let $\varepsilon_{n}$ be a sequence of positive numbers with $\lim _{n \rightarrow \infty} \varepsilon_{n}=0$. Let $r_{n}$ be an increasing sequence of positive numbers with $\lim _{n \rightarrow \infty} r_{n}=1$. Suppose that $r_{n}+\varepsilon_{n}<r_{n+1}$. Let $r(\theta)$ be an arbitrary increasing continuous function, $0 \leq \theta<\infty$, with the exceptions that

$$
r(\theta)= \begin{cases}r_{n}, & (4 n-2) \pi \leq \theta \leq(4 n-2) \pi+\frac{\pi}{4} \\ r_{n}+\varepsilon_{n}, & 4 n \pi \leq \theta \leq 4 n \pi+\frac{\pi}{4} .\end{cases}
$$

Let $S=\left\{r(\theta) e^{i \theta} \mid 0 \leq_{i}^{*} \theta<\infty\right\}$ be a spiral in $D$ and suppose $f \in \mathscr{V}(S)$. If $k(t)$ is defined in terms of $S$, then

$$
\pi \int_{2 \pi}^{4 n \pi+\pi / 4} \frac{d t}{k(t)} \geq \frac{\pi^{2}}{4} \sum_{i=1}^{n} \frac{1}{\varepsilon_{n}}=M_{n} .
$$

Given $\varepsilon>0$ there exists by Theorem 1 an $N$ such that for $n \geq N$,

$$
K\left(4 n \pi+\frac{\pi}{4}\right) \geq \exp \left\{\exp (1-\varepsilon) M_{n}\right\}
$$

By the arbitrariness of $r(\theta)$ in the intervals $4 n \pi+\pi / 4 \leq \theta \leq(4 n+2) \pi$ and the arbitrariness of the sequence $\varepsilon_{n}$ one may construct a spiral which grows extremely rapidly to $|z|=1$ while $M_{n}$, and hence $K(\theta)$, is forced to grow extremely rapidly.

\section{Section 3. Ahlfors-Denjoy Theorem in $\mathscr{V}(S)$.}

The methods Ahlfors used to prove the Denjoy conjecture are used here to prove a similar result in $\mathscr{V}(S)$. The result is that an entire 
function which tends to $n$ different asymptotic values on $n$ asymptotic paths has order exceeding $2 n$. See Theorem 2 for the comparable result in $\mathscr{V}(S)$.

Some preliminary facts are needed.

LeMma 2. [1, pp. 20,21]. Let $T_{1}, T_{2}, \cdots, T_{n-1}$ be $n-1$ continuous simple paths lying in $H: \mathscr{I}(\zeta)>0$ each of which connects the point 0 to $\infty$. Suppose $T_{i} \cap T_{j}=\{0\}$ if $i \neq j$. The set $H-\bigcup_{i=1}^{n-1} T_{i}$ is an open set which consists of $n$ simply connected components $\Omega_{1}, \Omega_{2}, \cdots, \Omega_{n}$. Let $\zeta=\varphi_{j}\left(z^{\prime}\right), j=1,2, \cdots, n$, be $n$ conformal transformations each of which maps the half-plane $\mathscr{I}\left(z^{\prime}\right)>0$ onto $\Omega_{j}$ such that $\varphi_{j}(0)=0$ and $\varphi_{j}(\infty)=\infty$. Then, given $\delta>0$, there exists among the numbers $1,2, \cdots, n$ an index
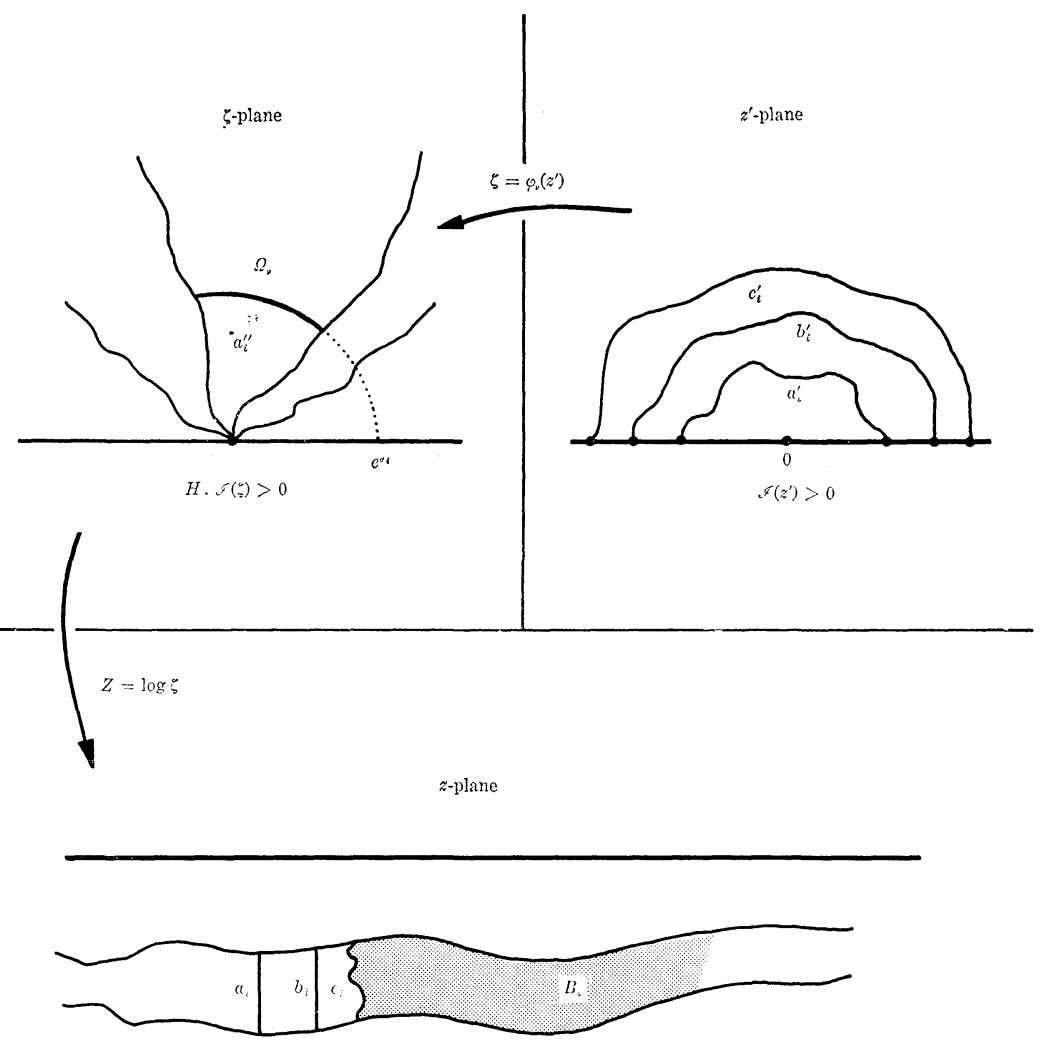

$U_{1}\left(\theta_{i}\right)$

K. $0<. q(Z)<$

Fig. 2 
$\nu$ and a sequence $\left\{a_{i}^{\prime}\right\}$ of simple arcs lying in the upper half-plane $\mathscr{I}\left(z^{\prime}\right)$ $>0$ which possess the following properties. Each $a_{i}^{\prime}$ connects a point of the positive real $z^{\prime}$-axis to a point of the negative real $z^{\prime}$-axis. As $i$ tends to $\infty$ the sets $a_{i}^{\prime}$ tend uniformly to $\infty$. For each $i$ and $z^{\prime} \in a_{i}^{\prime}$,

$$
\left|\varphi_{\nu}\left(z^{\prime}\right)\right|<\left|z^{\prime}\right|^{1 /(n+\delta)} .
$$

Figure 2 gives a picture of the situation in Lemma 2 and is referred to in the proof of Theorem 2.

Although the proof which is essentially the same as that given by Ahlfors [1] shall be omitted, some of the notation is presented for the computations which follow. For a given $\delta>0$ there exists the corresponding $\nu$ from Lemma 2. The function $Z=\log \zeta$ mapping $H: \mathscr{I}(\zeta)>0$ onto the strip $K: 0<\mathscr{I}(Z)<\pi$, also maps the region $\Omega_{\nu}$ of the lemma onto a simply connected strip region in $K$ which will be denoted by $B_{\nu}$. The sequence of sets $\left\{a_{i}^{\prime}\right\}$ in $\mathscr{I}\left(z^{\prime}\right)>0$ determined by the given $\delta$ and defined in the statement of Lemma 2 correspond (in the proof of the lemma) under the mapping $Z=\log \varphi_{\nu}\left(z^{\prime}\right)$ to a sequence of vertical straight line crosscuts of $B_{v}$, each of which separates the point $+\infty$ from the point $-\infty$ in the sense of Ahlfors [1]. The crosscut corresponding to $a_{i}^{\prime}$ will be denoted by $a_{i}$. The segment $a_{i}$ lies above a point on the real axis which will be denoted by $\sigma_{i}$ (See Figure 2). That is for each $i$

$$
\sigma_{i}=\mathscr{R}(Z), \quad Z \in a_{i} .
$$

The proof of Lemma 2 shows that

$$
\lim _{i \rightarrow \infty} \sigma_{i}=\infty .
$$

THEOREM 2. Let $f(z)$ be holomorphic in $D, S_{1}, S_{2}, \cdots, S_{n}$ be spirals in $D$ as defined in Section 1 , and let $a_{1}, a_{2}, \cdots, a_{n}$, be distinct finite complex numbers. If $f(z) \rightarrow a_{i}$ as $|z| \rightarrow 1$ with $z \in S_{i}, i=1,2, \cdots, n$, then $\rho\left(S_{i}\right) \geq n, i=1,2, \cdots, n$.

Proof. It may be assumed that $S_{i} \cap S_{j}=\{0\}$ if $i \neq j$. Assume the contrary of the theorem for some spiral which is taken to be $S=S_{n}$.

Define $\varphi(\theta), z=g(\zeta), k(\theta), P_{\theta}, Q_{\theta}$ and $U_{1}(\theta)$ as in Section 1 with respect to the spiral $S=S_{n}$.

According to the proof of Theorem 1 there exists a $\theta_{0}$ such that for all sufficiently large $\theta$, 


$$
U_{1}(\theta)>\pi \int_{\theta_{0}}^{\theta} \frac{d t}{\varphi(t)} .
$$

By the mapping $z=g(\zeta)$ the spirals $S_{1}, S_{2}, \cdots, S_{n-1}$ are transformed into sets $T_{1}, T_{2}, \cdots, T_{n-1}$ in $H: \mathscr{I}(\zeta)>0$ which satisfy the hypotheses of Lemma 2. Let $\delta$ be any positive number.

According to the lemma there is a sequence $\left\{a_{i}^{\prime}\right\}$ and $a \nu \in\{1,2, \cdots, n\}$ such that the inequalities (9) hold. There is also the sequence $\left\{\sigma_{i}\right\}$ of real numbers with $\lim _{i \rightarrow \infty} \sigma_{i}=\infty$ satisfying (10).

Consider the function

$$
G(\theta)=\pi \int_{\theta_{0}}^{\theta} \frac{d t}{\varphi(t)}
$$

$G(\theta)$ is a continuous increasing function with $\lim _{\theta \rightarrow \infty} G(\theta)=\infty$. By the intermediate value theorem, there exists a sequence $\left\{\theta_{i}\right\}$ with $\lim _{i \rightarrow \infty} \theta_{i}=\infty$ such that for some positive integer $N_{1}$ and $i \geq N_{1}$,

$$
G\left(\theta_{i}\right)=\pi \int_{\theta_{0}}^{\theta_{i}} \frac{d t}{\varphi(t)}=\sigma_{i}
$$

With each $\theta_{i}$, there are the sets $k_{\theta_{i}}, P_{\theta_{i}}$, and $Q_{\theta_{i}}$ in $D^{\prime}, H$ and $K: 0<\mathscr{I}(Z)<\pi$, respectively. Now the crosscut $a_{i}$ of $B_{\nu}$ above $\sigma_{i}$ lies in $B_{\nu}$ which lies in $K$. The reader is referred to Figures 1 and 2. Define $c_{i}=Q_{\theta_{i}} \cap B_{\nu}$ and $b_{i}$ to be the straight line crosscut of $B_{\nu}$ above the real number $U_{1}\left(\theta_{i}\right)$ of (4). By definition of $U_{1}\left(\theta_{i}\right), c_{i}$ lies entirely to the right of $b_{i}$. But by (12) and (13), $a_{i}$ lies to the left of $b_{i}$. Under the mapping $z^{\prime}=\varphi_{\nu}^{-1}\left(e^{Z}\right), a_{i}^{\prime}$ is the image of $a_{i}$ as mentioned above. Let $b_{i}^{\prime}$ and $c_{i}^{\prime}$ be the images of $b_{i}$ and $c_{i}$, respectively, under this same mapping. The set $b_{i}^{\prime}$ divides the half-plane $\mathscr{I}\left(z^{\prime}\right)>0$ into regions $H_{1}$ and $H_{2}$ which are bounded and unbounded, respectively. By the normalization of the conformal transformations, $a_{i}^{\prime}$ lies in $H_{1}$ and $c_{i}^{\prime}$ lies in $\mathrm{H}_{2}$.

Define $w=F\left(z^{\prime}\right)=f\left(g\left(\varphi_{\nu}\left(z^{\prime}\right)\right)\right), \mathscr{I}\left(z^{\prime}\right) \geq 0$, where $\varphi_{\nu}\left(z^{\prime}\right)$ is to be taken as the extension of the mapping of $\mathscr{I}\left(z^{\prime}\right)>0$ into $H$ to the boundary. The function $F\left(z^{\prime}\right)$ tends to different limits as $z^{\prime} \rightarrow+\infty$ and $z^{\prime} \rightarrow-\infty$ with $z^{\prime}$ real by the hypotheses of the theorem so that by a well known theorem of Lindelöf it is unbounded in $\mathscr{I}\left(z^{\prime}\right)>0$. This unboundedness is what shall be contradicted.

Since $a_{i}^{\prime}$ tends to infinity uniformly by Lemma 2 , it is seen that 
$\sup _{z^{\prime} \in a_{i}^{\prime}}\left|F\left(z^{\prime}\right)\right|$ exceeds the bound of $\left|F\left(z^{\prime}\right)\right|$, for $z^{\prime}$ on the real axis, provided $i$ exceeds some positive integer $N_{2}$. By the maximum principle, for $i \geq \max \left(N_{1}, N_{2}\right)$,

$$
\operatorname{Max}_{z^{\prime} \in a_{i}^{\prime}}\left|F\left(z^{\prime}\right)\right| \leq \operatorname{Max}_{z^{\prime} \in b_{i}^{\prime}}\left|F\left(z^{\prime}\right)\right| \leq \operatorname{Max}_{z^{\prime} \in c_{i}^{\prime}}\left|F\left(z^{\prime}\right)\right| .
$$

Since $c_{i} \subset Q_{\theta_{i}}$

$$
\operatorname{Max}_{z^{\prime} \in c_{i}^{\prime}}\left|F\left(z^{\prime}\right)\right| \leq \operatorname{Max}_{z \in Q_{i}}\left|F\left(z^{\prime}\right)\right|=\operatorname{Max}_{z \in k_{\theta_{i}}}|f(z)|=K\left(\theta_{i}\right) .
$$

Thus

$$
\operatorname{Max}_{z^{\prime} \in a_{i}^{\prime}}\left|F\left(z^{\prime}\right)\right| \leq K\left(\theta_{i}\right), \quad \text { if } i \geq \max \left(N_{1}, N_{2}\right) .
$$

By assumption, $\rho(S)=A<n$, and by (3) of Lemma 1, $k(t)$ may be replaced by $\varphi(t)$. The value of $\theta_{0}$ obtained in inequality (12) may be used in the definition of $\rho(S)$. Then

$$
\varlimsup_{\theta \rightarrow \infty} \frac{\log \log K(\theta)}{\pi \int_{\theta_{0}}^{\theta} \frac{d t}{\varphi(t)}}=\rho(S)=A<n .
$$

Therefore, given any $\varepsilon<0$, there exists an $N_{3}$ such that if $i \geq N_{3}$, then

$$
K\left(\theta_{i}\right) \leq \exp \left\{\exp \left[(A+\varepsilon) \pi \int_{\theta_{0}}^{\theta_{i}} \frac{d t}{\varphi(t)}\right]\right\}
$$

Let $z^{\prime} \in a_{i}^{\prime}$ be an arbitrary point and let $N=\operatorname{Max}\left(N_{1}, N_{2}, N_{3}\right)$. Applying inequalities (14), (16), and (12) together with (13), for $i \geq N$ one has

$$
\left|F\left(z^{\prime}\right)\right| \leq \exp \left[\exp \sigma_{i}\right]^{A+\varepsilon}
$$

Let $a_{i}^{\prime \prime}$ be the image in $\Omega_{\nu}$ of $a_{i}^{\prime}$ under the mapping $\zeta=\varphi_{\nu}\left(z^{\prime}\right)$ (See Figure 2). Then $a_{i}^{\prime \prime}$ is also the image of the straight line segment $a_{i}$ under the mapping $\zeta=e^{Z}$. Since $\mathscr{R}(Z)=\sigma_{i}$ for $Z \in a_{i}$, one obtains for the arbitrary point $z^{\prime} \in \alpha_{i}^{\prime}$,

$$
\left|\varphi_{\nu}\left(z^{\prime}\right)\right|=\exp \sigma_{i}
$$

That is for $i \geq N$, (17) becomes

$$
\left|F\left(z^{\prime}\right)\right| \leq \exp \left\{\left|\varphi_{\nu}\left(z^{\prime}\right)\right|^{A+\varepsilon}\right\} .
$$


But Lemma 2, inequality (9), holds for $z^{\prime} \in a_{i}$ and $\varphi_{\nu}\left(z^{\prime}\right)$. Hence, for $i \geq N$,

$$
\left|F\left(z^{\prime}\right)\right| \leq \exp \left|z^{\prime}\right|^{(1 / n+\delta)(A+\varepsilon)} .
$$

Since $z^{\prime} \in a_{i}^{\prime}$ was an arbitrary choice, the inequality (19) holds for every $z^{\prime} \in \alpha_{i}^{\prime}$, provided only that $i \geq N$.

Since $A<n$, choose $\varepsilon$ and $\delta$ such that $(A+\varepsilon)(1 / n+\delta)=\gamma<1$. Then one obtains the sequence $\left\{a_{i}^{\prime}\right\}$ and the $N$ such that

$$
\left|F\left(z^{\prime}\right)\right| \leq \exp \left|z^{\prime}\right|^{\gamma}, \quad z^{\prime} \in a_{i}^{\prime}, \quad i \geq N .
$$

Since $\left|F\left(z^{\prime}\right)\right|$ is bounded on the real axis, it is a bounded function by the Phragmén-Lindelöf Principle. But $F\left(z^{\prime}\right)$ has already been shown to be unbounded. This contradiction establishes Theorem 2 .

\section{Section 4. MacIntyre's extension in $\mathscr{V}(S)$.}

MacIntyre [4] proves the following theorem: If $f(z)$ is entire and is bounded on $n$ disjoint arcs each connecting zero to infinity and if $f$ is unbounded in all the regions bounded by these arcs then the lower order of $f$ exceeds $n / 2$. Theorem 3 is the comparable result in $\mathscr{V}(S)$.

THEOREM 3. If $f(z)$ is in $\mathscr{V}\left(S_{i}\right), i=1,2, \cdots, n$ where $S_{1}, S_{2}, \cdots, S_{n}$ are spirals in $D$ such that $S_{i} \cap S_{j}=\{0\}$ if $i \neq j$, and if $f(z)$ is unbounded in each of the regions of $D-\cup_{i=1}^{n} S_{i}$, then

$$
\lambda\left(S_{i}\right) \geq n \quad i=1,2, \cdots, n .
$$

The proof of this theorem, which follows Lemmas 3 and 4, uses results of Ahlfors and MacIntyre.

LEMMA 3. [4]. Let $f(z)$ be holomorphic and $|f(z)| \leq M$ in a simply connected region $G$ which is contained in the sector $0 \leq \arg z \leq \pi / p$, $|z| \leq R$, where $p>1 / 2$. Suppose that part of the boundary of $G$ is on $|z|=R$ and that $|f(z)| \leq m$ in some neighborhood of each point of the part of the boundary of $G$ which is in $|z|<R$. Then for any point $r e^{i \theta}$ in $G$,

$$
\begin{aligned}
& \log \left|f\left(r e^{i \theta}\right)\right| \leq 2 / \pi\left\{\log m \operatorname{Arctan} \frac{1-(r / R)^{2 p}}{2(r / R)^{p}}\right. \\
& \left.+\log M \operatorname{Arctan} \frac{2(r / R)^{p}}{1-(r / R)^{2 p}}\right\}
\end{aligned}
$$


MacIntyre proves this lemma for the case $R=1$ in the reference cited. The lemma is obtained by considering $f(R w)$ in $|w| \leq 1$.

Suppose $T_{1}, T_{2}, \cdots, T_{n+1}$ are in $\bar{H}: \mathscr{I}(\zeta) \geq 0$, where $T_{2}, T_{3}, \cdots, T_{n}$ are simple arcs each of which connects 0 to $\infty, T_{1}$ is the positive real axis, $T_{n+1}$ is the negative real axis, and $T_{i} \cap T_{j}=\{0\}, i \neq j$. Then $H-\bigcup_{\nu=2}^{n} T_{\nu}$ is open and has $n$ simply connected components $\Omega_{1}, \Omega_{2}, \cdots, \Omega_{n}$. Fix the order by supposing that $T_{\nu} \cup T_{\nu+1}$ bounds $\Omega_{\nu}, \nu=1,2, \cdots, n$. The reader is referred to Figure 3. Let $\nu$ be any of the numbers $1,2, \cdots, n$. Given $R>1, T_{\nu}$ has a last intersection with $|\zeta|=1$ and a first intersection with $|\zeta|=R$ which shall be denoted by $A_{\nu}$ and $B_{\nu}$, respectively. Let $T_{\nu}^{\prime}$ be that part of $T_{\nu}$ between $A_{\nu}$ and $B_{\nu}, \gamma_{\nu}$ the arc of $|\zeta|=1$ between $A_{\nu}$ and $A_{v+1}$ (in $H$ ), and $\Gamma_{\nu}$ the arc of $|\zeta|=R$ between $B_{v}$ and $B_{v+1}$ (in $H$ ). Denote by $D_{\nu}(R)$ the region bounded by $T_{\nu}^{\prime} \cup \Gamma_{\nu} \cup T_{\nu+1}^{\prime} \cup \gamma_{\nu}$.

By means of conformal transformations and the Schwarz-Christoffel

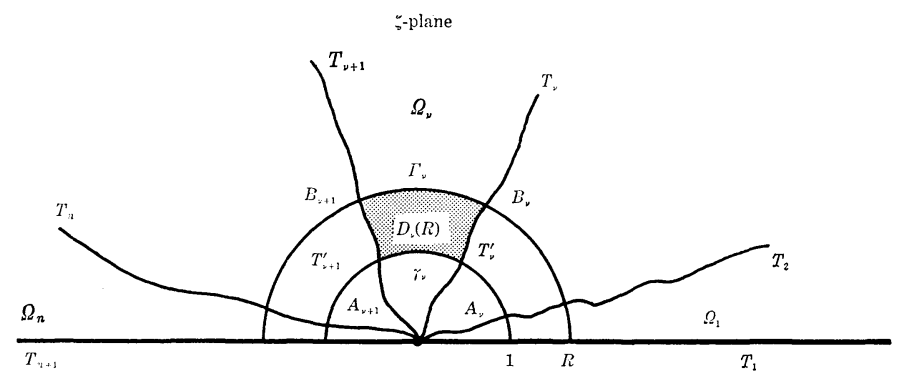

$H: \mathscr{I}(\zeta)>0$

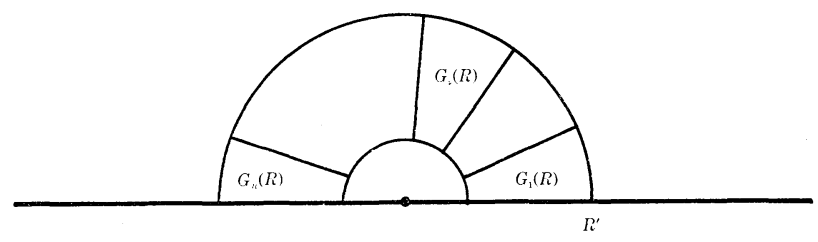

$f(x)>0$

Fig. 3 
transformation the region $D_{\nu}(R)$ can be mapped onto a set $G_{\nu}(R)$ which is a sector of the annulus $1 \leq|w| \leq R^{\prime}$ for some $R^{\prime}$. The arc $\Gamma_{\nu}$ can be made to correspond to that part of the boundary of $G_{\nu}(R)$ which lies on $|w|=R^{\prime}$. The sets $G_{\nu}(R), \nu=1,2, \cdots, n$, can be arranged so that they do not overlap and the union of their closures is $1 \leq|w| \leq R^{\prime}$, $\mathscr{I}(w) \geq 0$. See MacIntyre [4] for details. The $n$ conformal transformations so obtained shall be denoted as one by $\zeta=\psi_{R}(w)$. The number $R^{\prime}$ will henceforth be referred to as the number associated with $R$.

LEMMA 4. Given $R>1$, the number $R^{\prime}$ associated with $R$ satisfies

$$
R^{\prime} \geq R
$$

A proof of this lemma and a discussion of the formation of the sets $G_{\nu}(R)$ above can be found in MacIntyre [4].

Proof of Theorem 3. It is first proved that for any $\theta_{0}>2 \pi$,

$$
\lim _{\theta \rightarrow \infty} \frac{\log K(\theta)}{\exp \left[n \pi \int_{\theta_{0}}^{\theta} \frac{d t}{\varphi(t)}\right]}>0
$$

where $K(\theta)$ and $\varphi(\theta)$ are defined in terms of any one of the spirals $S_{1}, S_{2}, \cdots, S_{n}$. Assume that this inequality is false for some $\theta_{0}>2 \pi$ and some $S_{i}$ which is taken for convenience to be $S=S_{1}$. Then there is a sequence $\left\{\varepsilon_{i}\right\}$ with $\varepsilon_{i}>0$ and $\lim _{i \rightarrow \infty} \varepsilon_{i}=0$, and a sequence $\left\{\theta_{i}\right\}$ with $\lim _{i \rightarrow \infty} \theta_{i}=\infty$ such that

$$
\log K\left(\theta_{i}\right) \leq \varepsilon_{i} \exp \left[n \pi \int_{\theta_{0}^{\prime}}^{\theta_{i}} \frac{d t}{\varphi(t)}\right], \quad i=1,2, \cdots
$$

where $K(\theta)$ and $\varphi(\theta)$ are defined as usual relative to $S$. With $z=g(\zeta)$ defined as in Section 1 relative to $S, P_{\theta_{i}}$ is the image of the $k_{\theta_{i}}$ in $H$ : $\mathscr{I}(\zeta)>0$ under $g$. By Ahlfors' Theorem [1] again, there exists $\theta_{0}^{\prime}>\theta_{0}$ such that if $i$ is sufficiently large say $i \geq N$, then $P_{\theta_{i}}$ lies outside the circle

$$
C_{i}:|\zeta|=\exp \left[\pi \int_{\theta_{0}^{\prime}}^{\theta_{i}} \frac{d t}{\varphi(t)}\right]
$$

Setting 


$$
\varepsilon_{i}^{\prime}=\varepsilon_{i} \exp \left[\pi \int_{\theta_{0}}^{\theta_{0}^{\prime}} \frac{d t}{\varphi(t)}\right], \quad i=1,2, \cdots,
$$

and

$$
R_{i}=\exp \left[\pi \int_{\theta_{0}^{\prime}}^{\theta_{i}} \frac{d t}{\varphi(t)}\right], \quad i \geq N
$$

inequalities (23) becomes

$$
\log K\left(\theta_{i}\right) \leq \varepsilon_{i}^{\prime} R_{i}^{n}, \quad i \geq N .
$$

During the proof of this theorem there will be several occasions when it is convenient to extract subsequences having certain properties from the original sequence $\left\{\theta_{i}\right\}$. Each such subsequence has the limit infinity and this is the only property which is essential to the proof. Thus to keep the notation simple, it shall always be assumed that the subsequence is renumbered to be the original sequence. For example, $P_{\theta_{i}}$ lies outside $C_{i}$ and inequalities (26) shall hold for every $i$. Under $z=g(\zeta)$ the spirals $S_{1}, S_{2}, S_{3}, \cdots, S_{n}$ correspond to $n+1$ paths in $\bar{H}$ which satisfy Lemma 3 . With the notation of Lemma 4, for each $R_{i}>1$ of (25) there is the associated $R_{i}^{\prime}$ which, by (21), satisfies

$$
R_{i}^{\prime} \geq R_{i}, \quad i=1,2, \cdots .
$$

For $i$ fixed, consider the sets $G_{\nu}(R), \nu=1,2, \cdots, n$, defined in the discussion following Lemma 3 . These sets lie in the half annulus $1 \leq$ $|w| \leq R_{i}^{\prime}, \mathscr{I}(w) \geq 0$. Since each of the $n$ sets $G_{\nu}(R)$ is a sector of this half annulus, there is a $\nu_{i}$ such that $G_{\nu_{i}}\left(R_{i}\right)$ lies in a sector of opening smaller than or equal to $\pi / n$.

As $i$ varies, a sequence $\left\{\nu_{i}\right\}$ is obtained, each number of which is selected from the finite set $\{1,2, \cdots, n\}$. Thus there is among these numbers an index $\nu^{\prime}$ and a subsequence $\left\{i_{k}\right\}$ of $\{i\}$, such that each $G_{\nu^{\prime}}\left(R_{i_{k}}\right)$ lies in a sector of opening less than or equal to $\pi / n$. As decided above, it is assumed that $G_{\nu^{\prime}}\left(R_{i}\right)$ has this property for every $i$. With this $\nu^{\prime}$, consider $\Omega_{\nu^{\prime}}$ and the function $f(g(\zeta))$ restricted to $\Omega_{\nu^{\prime}}$. This function is bounded on the boundary of $\Omega_{\nu^{\prime}}$ and bounded in $|\zeta| \leq 1$. Without loss of generality it can be assumed that the bound in both cases is 1 .

With each $R_{i}$, there is the "function" $\zeta=\psi_{R_{i}}(w)$ which maps the region $G_{\nu^{\prime}}\left(R_{i}\right)$ onto the region $D_{\nu^{\prime}}\left(R_{i}\right)$. For simplicity let $G_{i}=G_{\nu^{\prime}}\left(R_{i}\right)$, $D_{i}=D_{\nu^{\prime}}\left(R_{i}\right)$ and $\chi_{i}=\psi_{R_{i}}$, since $\nu^{\prime}$ is now fixed. 
Define $F_{i}(w)=f\left(g\left(\chi_{i}(w)\right)\right), w \in G_{i}, i=1,2, \cdots$. It should be recalled that the boundary arc of $G_{i}$ on $|w|=R_{i}^{\prime}$ corresponds under $\zeta=\psi_{i}(w)$ to the boundary arc $\Gamma_{\nu^{\prime}}$, of $D_{i}$ on $|\zeta|=R_{i}$. Hence, for the remaining part of the boundary of $G_{i}$,

$$
\left|F_{i}(w)\right| \leq 1
$$

Moreover,

$$
\sup _{|w|=R_{i}^{\prime}}\left|F_{i}(w)\right|=\sup _{\substack{|\zeta|=R_{i} \\ \zeta \in D_{i}}}|f(g(\zeta))|
$$

and

$$
K\left(\theta_{i}\right)=\sup _{\zeta \in P \theta_{i}}|f(g(\zeta))|
$$

The set $P_{\theta_{i}}$ lies outside of $|\zeta|=R_{i}$, by definition (25) of $R_{i}$ and application of Ahlfors' Theorem. Moreover $\sup _{\substack{|w|=R_{i} \\ w \in \bar{G}_{i}}}\left|F_{i}(w)\right|$ exceeds 1 for all sufficiently large $i$ (assume these facts for every $i$ ). Thus it follows from (28) and (29) that

$$
\sup _{\substack{|w|=R_{i} \\ w \in \bar{G}_{i}}}\left|F_{i}(w)\right| \leq K\left(\theta_{i}\right)
$$

Let $\zeta_{0} \in \Omega_{\nu^{\prime}}$, with $\left|\zeta_{0}\right|>1$. With $G=G_{i}, p=n, R=R_{i}^{\prime}, m=1$, and $M=K\left(\theta_{i}\right)$ apply Lemma 3 . Inequality (20) becomes

$$
\log \left|F_{i}\left(w_{i}\right)\right| \leq \frac{2}{\pi} \log K\left(\theta_{i}\right) \operatorname{Arctan} \frac{2\left(\left|w_{i}\right| / R_{i}^{\prime}\right)^{n}}{1-\left(\left|w_{i}\right| / R_{i}^{\prime}\right)^{2 n}},
$$

where $w_{i}$ is defined to be the image of $\zeta_{0}$ in $G_{i}$, under $\zeta=\psi_{i}(w)$. That the sequence $w_{i}$ has a bounded subsequence can be shown using normal families. It is, of course, assumed that

$$
\left|w_{i}\right| \leq M_{0}<\infty, \quad i=1,2, \cdots
$$

There is an $M_{1}<\infty$ such that

$$
\log \left|F_{i}\left(w_{i}\right)\right| \leq M_{1} \log K\left(\theta_{i}\right) R_{i}^{\prime-n} .
$$

But $R_{i}^{\prime} \geq R_{i}$ so that (31) becomes

$$
\log \left|F_{i}\left(w_{i}\right)\right| \leq M_{1} \log K\left(\theta_{i}\right) R_{i}^{-n} .
$$

Using inequality (26) and equality (25) of the definition of $R_{i}$, it follows that for every $i$, 


$$
\log \left|F_{i}\left(w_{i}\right)\right| \leq M_{1} \varepsilon_{i}^{\prime} .
$$

But $F_{i}\left(w_{i}\right)=f\left(g\left(\zeta_{0}\right)\right)$ is a constant which by (33) must satisfy $\left|f\left(g\left(\zeta_{0}\right)\right)\right| \leq 1$ since $\lim _{i \rightarrow \infty} \varepsilon_{i}^{\prime}=0$. This means that $f(g(\zeta))$ is bounded in $\Omega_{\nu^{\prime}}$. Clearly, from the hypotheses of the theorem, $f(g(\zeta))$ is not bounded in $\Omega_{\nu^{\prime}}$. This contradiction yields (22).

It has now been established, that for some $a>0$,

$$
\lim _{\theta \rightarrow \infty} \frac{\log K(\theta)}{\exp \left[n \pi \int_{\theta_{0}}^{\theta} \frac{d t}{\varphi(t)}\right]}>a>0,
$$

with $K(\theta)$ and $\varphi(\theta)$ defined for any of the spirals $S_{i}$ in the statement of Theorem 3 and $\theta_{0}>2 \pi$ arbitrary. Thus, for large enough $\theta$,

$$
\log \log K(\theta)>\log a+n \pi \int_{\theta_{0}}^{\theta} \frac{d t}{\varphi(t)},
$$

or,

$$
\frac{\log \log K(\theta)}{\pi \int_{\theta_{0}}^{\theta} \frac{d t}{k(t)}}>\frac{\log a}{\pi \int_{\theta_{0}}^{\theta} \frac{d t}{k(t)}}+n \frac{\pi \int_{\theta_{0}}^{\theta} \frac{d t}{\varphi(t)}}{\pi \int_{\theta_{0}}^{\theta} \frac{d t}{k(t)}}
$$

By (3) of Lemma 1, it follows that

$$
\lambda\left(S_{i}\right)=\lim _{\theta \rightarrow \infty} \frac{\log \log K(\theta)}{\pi \int_{\theta_{0}}^{\theta} \frac{d t}{k(t)}} \geq n,
$$

where $k(t)$ and $K(\theta)$ are defined relative to $S_{i}, i=1,2, \cdots, n$. This completes the proof of Theorem 3 .

COROLLARY. Theorem 3 implies both Theorem $1(n=1)$ and Theorem 2.

It should be remarked that for $f \in \mathscr{V}(S)$ neither $\rho(S)$ nor $\lambda(S)$ are guaranteed to be finite. It is expected that there are functions for which they are finite for two reasons. The first reason is that the "right" results are obtained and the second reason is that the estimates of the Phragmén-Lindelöf principle and Ahlfors' Theorem may be asymptotically sharp in the case of some spirals. By right results is meant the analogies between class $\mathscr{V}(S)$ and entire functions. 


\section{REFERENCES}

[1] Ahlfors, L., Untersuchungen zur Theorie der konformen Abbildung und der ganzen Functionen, Acta Societatis Scientiarum Fennicae, Nova Series A, vol. 1 (1930).

[2] Bagemihl, F., and Seidel, W., Koebe arcs and Fatou points of normal functions, Comment. Math. Helv., 36 (1961).

[ 3 ] Boas, R., Entire Functions, Academic Press Inc., New York, N.Y., (1954).

[ 4 ] MacIntyre, A., On the asymptotic paths of integral functions of finite order, Journal London Mathematical Society, vol. 10 (1935), pp. 34-39.

[5] Phragmén, E., Lindelöf, E., Sur une extension d'un principe classique de l'Analyse et sur quelques propriétés des functions monogénes dans le voisinage d'un point singulier, Acta Mathematica, vol. 31 (1908), pp. 381-406.

[6] Schnitzer, F., and Seidel, W., On the modulus of unbounded holomorphic functions, Mathematische Zeitschrift, vol. 88 (1965), pp. 301-308.

[7] Valiron, G., Sur les singularités de certains functions holomorphes et de leurs inverses, Journal de Mathématiques pures et appliquées (9), vol. 15 (1936), pp. 432-435.

Fordham University 